\title{
Absolute instability modes due to rescattering of stimulated Raman scattering in a large nonuniform plasma
}

\author{
Yao Zhao ${ }^{1}$, Zhengming Sheng ${ }^{2,3,4,5}$, Suming Weng ${ }^{2,3}$, Shengzhe $\mathrm{Ji}^{1,6}$, and Jianqiang Zhu ${ }^{1,3}$ \\ ${ }^{1}$ Key Laboratory of High Power Laser and Physics, Shanghai Institute of Optics and Fine Mechanics, Chinese Academy of Sciences, \\ Shanghai 201800, China \\ ${ }^{2}$ Key Laboratory for Laser Plasmas (MoE), School of Physics and Astronomy, Shanghai Jiao Tong University, Shanghai 200240, China \\ ${ }^{3}$ Collaborative Innovation Center of IFSA (CICIFSA), Shanghai Jiao Tong University, Shanghai 200240, China \\ ${ }^{4}$ SUPA, Department of Physics, University of Strathclyde, Glasgow G4 ONG, UK \\ ${ }^{5}$ Tsung-Dao Lee Institute, Shanghai Jiao Tong University, Shanghai 200240, China \\ ${ }^{6}$ University of Chinese Academy of Sciences, Beijing 100049, China \\ (Received 8 November 2018; revised 16 January 2019; accepted 29 January 2019)
}

\begin{abstract}
Absolute instability modes due to secondary scattering of stimulated Raman scattering (SRS) in a large nonuniform plasma are studied theoretically and numerically. The backscattered light of convective SRS can be considered as a pump light with a finite bandwidth. The different frequency components of the backscattered light can be coupled to develop absolute SRS instability near their quarter-critical densities via rescattering process. The absolute SRS mode develops a Langmuir wave with a high phase velocity of about $c / \sqrt{3}$ with $c$ the light speed in vacuum. Given that most electrons are at low velocities in the linear stage, the absolute SRS mode grows with very weak Landau damping. When the interaction evolves into the nonlinear regime, the Langmuir wave can heat abundant electrons up to a few hundred keV via the SRS rescattering. Our theoretical model is validated by particle-in-cell simulations. The absolute instabilities may play a considerable role in the experiments of inertial confinement fusion.
\end{abstract}

Keywords: laser plasma interactions; stimulated Raman scattering; two plasmon decay instability; hot electron

\section{Introduction}

Stimulated Raman scattering (SRS), the decay of incident laser into scattered light and electron plasma wave ${ }^{[1-3]}$, remains to be one of the major obstacles to direct-drive, indirect-drive and possible hybrid-drive schemes ${ }^{[4-9]}$. SRS generates a large amount of hot electrons ${ }^{[10-12]}$, which can preheat the capsule. This has significant impacts on the implosion and ignition processes ${ }^{[13,14]}$. Even though there have been tremendous studies on the problem of hot electron production via SRS both in theory and experiments ${ }^{[3,15]}$, the generation mechanisms of hot electrons at nonlinear stages involving multiple driving waves are not yet fully understood.

Rescattering of SRS has been reported both theoretically and experimentally in different parametric conditions ${ }^{[16-20]}$. Some previous works have discussed about the effects of

Correspondence to: Y. Zhao, No. 390 Qinghe Road, Jiading District, Shanghai 201800, China. Email: yaozhao@siom.ac.cn
SRS rescattering on the nonlinear scattering spectrum and saturation mechanism of instabilities in the regime where backward SRS is heavily damped ${ }^{[17,18]}$. Anomalously hot electrons due to rescattering of SRS have been studied recently ${ }^{[16]}$. However, this work mainly studied the rescattering of SRS in homogeneous plasma, i.e., the absolute instabilities induced by rescattering in inhomogeneous plasma not yet been sufficiently discussed. Generally, the absolute instability mode is the most significant instability in inhomogeneous plasma with the smallest threshold ${ }^{[2]}$. In addition, the phase velocity of the Langmuir wave excited by the absolute SRS is larger than that of other rescattering instability modes. In our work, we present a mechanism of hot electron production via the absolute instabilities in a large nonuniform plasma due to rescattering of SRS. It is well known that parametric instabilities in inhomogeneous plasma are convective in one-dimensional (1D) geometry except near the quarter-critical density, where both SRS and two plasmon decay (TPD) become absolute instabilities ${ }^{[2]}$. 
For the indirect-drive scheme, even though there is a large underdense plasma region, the absolute instability is usually ignored since the maximum density of the plasma is typically less than $0.2 n_{c}{ }^{[21]}$. In this work, we investigate the generation mechanism and associate conditions for the absolute instabilities. Our work suggests that the absolute instability modes induced via rescattering of SRS are the important mechanisms for hot electron production for long time interactions between laser and large scale inhomogeneous plasma, even if the maximum plasma density is less than $0.2 n_{c}$. Our theoretical model is supported by particlein-cell (PIC) simulations.

\section{Theoretical analysis of the absolute instability modes via rescattering of $\mathrm{SRS}$}

Here we consider cascaded scattering of SRS in an inhomogeneous plasma, including the first-order scattering occurring at the plasma electron density $n_{1}$, the subsequent second-order scattering at the density $n_{2}$ and the third-order scattering at the density $n_{3}$. The plasma has a positive density gradient along the laser propagation direction with an electron density range $\left[n_{\min }, n_{\max }\right]$, where $n_{\min }$ and $n_{\max }$ are the minimum and maximum densities, respectively. Assume that the corresponding electron plasma wave frequencies are given by $\omega_{p 1}=\sqrt{4 \pi n_{1} e^{2} / m_{e}}, \omega_{p 2}=\sqrt{4 \pi n_{2} e^{2} / m_{e}}$ and $\omega_{p 3}=\sqrt{4 \pi n_{3} e^{2} / m_{e}}$. According to the frequency match conditions for three wave coupling, the scattered light frequencies via the first-order scattering, second-order scattering and third-order scattering are given by $\omega_{s 1}=\omega_{0}-\omega_{p 1}$ (the corresponding wavenumber $k_{s 1} \leqslant 0$ ), $\omega_{s 2}=\omega_{0}-$ $\omega_{p 1}-\omega_{p 2}$ (the corresponding wavenumber $k_{s 2} \geqslant 0$ ) and $\omega_{s 3}=\omega_{0}-\omega_{p 1}-\omega_{p 2}-\omega_{p 3}$ (the corresponding wavenumber $\left.k_{s 3} \leqslant 0\right)$. The absolute instabilities in inhomogeneous plasma are found at the quarter-critical density of the incident electromagnetic wave. This can occur in certain regions in plasma via multiple SRS processes. Via multiple-order scattering, the incident laser energy is dissipated to plasma wave excitation and hot electron production.

In the following, we examine the absolute instabilities via the second-order and third-order scattering. With the first-order backward SRS developed at $n_{1}$ and the secondorder scattering at $n_{2}$, the second-order scattering becomes the absolute instability when it is developed at the quartercritical density of the first-order scattered light, i.e.,

$$
\omega_{s 1}=\omega_{s 2}+\omega_{L 2} \approx 2 \omega_{L 2}
$$

where $\omega_{L 2}=\sqrt{\omega_{p 2}^{2}+3 k_{L 2}^{2} v_{e}^{2}}$ with $v_{e}$ the electron thermal velocity. In the following, we take $\omega_{L 2}=\omega_{p 2}$ for simplicity by ignoring the plasma electron temperature. Since $n_{2} \leqslant n_{1}$, one finds that $n_{1} \geqslant n_{c} / 9$ and $n_{2} \leqslant n_{c} / 9$ from Equation (1). This indicates that the backscattering light from the region (a)

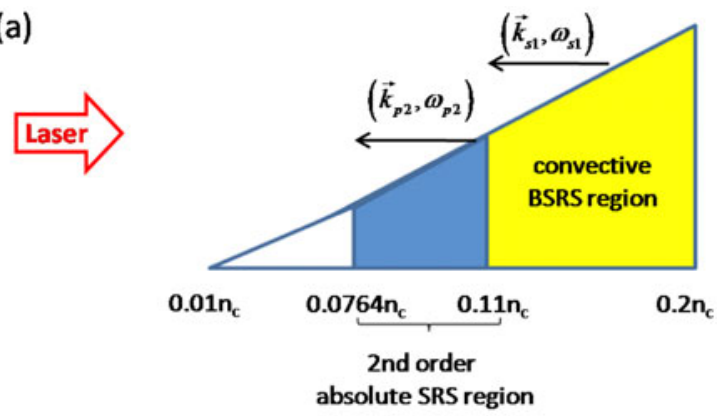

(b)

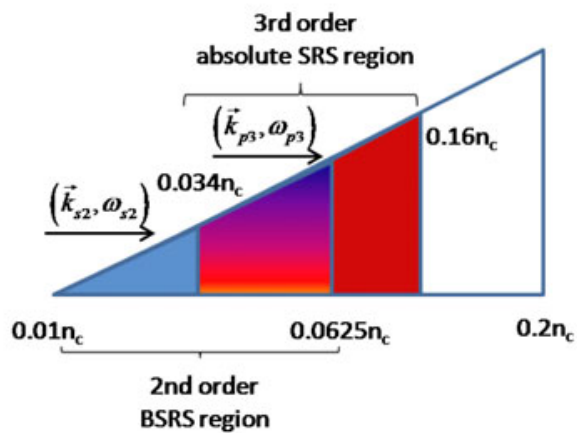

Figure 1. Schematic diagram for absolute instability regions due to (a) the second-order rescattering of SRS and (b) the third-order rescattering of SRS in a linearly inhomogeneous plasma with density $[0.01,0.2] n_{c}$. BSRS means backscattering of SRS.

$n_{1} \geqslant n_{c} / 9 \approx 0.11 n_{c}$ can trigger absolute instabilities in the density area $n_{2} \leqslant n_{c} / 9$. One may note that the first-order forward SRS light from $n_{1} \leqslant n_{c} / 9$ can induce absolute modes in the region $n_{2} \geqslant n_{c} / 9$. Therefore, the major difference between rescattering of forward and backward scattering lights is the density region for absolute instability. Without loss of generality, we mainly consider the rescattering of backward SRS in this paper.

With the condition $\omega_{s 1}=2 \omega_{p 2}$ or $1-\sqrt{n_{1} / n_{c}}=2 \sqrt{n_{2} / n_{c}}$ for the development of absolute instabilities, it is obvious that backscattered lights from different plasma density $n_{1}$ develop absolute modes at different density $n_{2}$. When the minimum quarter-critical density for the backscattering light $n_{2}^{\min }$ is within $\left[n_{\min }, n_{\max }\right]$, the absolute instability region is simply $\left[\left(1-\sqrt{n_{\max } / n_{c}}\right)^{2} / 4,1 / 9\right] n_{c}$. In this linear analysis, we ignored the coupling of backscattering lights from different plasma density between $n_{c} / 9$ and $n_{\max }$. As shown in Figure 1(a), for example, the initial plasma electron density is $[0.01,0.2] n_{c}$, and the minimum density for absolute SRS $n_{2}^{\min }=0.0764 n_{c}$ is found for backscattering from the density at $0.2 n_{c}$. The first-order convective SRS occurs in $[0.11,0.2] n_{c}$, and its backscattering light can induce absolute instabilities by second-order rescattering of SRS within $[0.0764,0.11] n_{c}$.

Analogously, assume that the first-order backward SRS develops at $n_{1}$, the second-order backward SRS develops at $n_{2}$, and the third-order backward SRS develops at $n_{3}$. 
If the third-order scattering is absolute, it is required that it is developed at its corresponding quarter-critical density, i.e.,

$$
\omega_{s 2}=\omega_{s 3}+\omega_{p 3} \approx 2 \omega_{p 3} .
$$

Since $n_{1} \geqslant n_{2}$ and $n_{3} \geqslant n_{2}$, one finds that $n_{2} \leqslant n_{c} / 16=$ $0.0625 n_{c}$ according to Equation (2). Therefore, the secondorder backward SRS developed in $\left[n_{\min }, 0.0625\right] n_{c}$ can induce the third-order absolute instability modes in a slightly higher density region. Note that $\omega_{0}=\omega_{p 1}+\omega_{p 2}+2 \omega_{p 3} \leqslant$ $\omega_{p 1}+3 \omega_{p 3}$ and that $\omega_{0}=\omega_{p 1}+\omega_{p 2}+2 \omega_{p 3} \geqslant 2 \omega_{p 2}+2 \omega_{p 3}$. One finds the absolute instability range of the third-order rescattering $\left[\left(1-\sqrt{n_{\max } / n_{c}}\right)^{2} / 9,\left(1-2 \sqrt{n_{\min } / n_{c}}\right)^{2} / 4\right] n_{c}$. The absolute instability region may be reduced by the upper or lower limits of the plasma density profile. As an example, for the given plasma electron density shown in Figure 1(b), the secondary backward SRS is developed in $[0.01,0.0625] n_{c}$, which can induce the third-order absolute instabilities in $[0.034,0.16] n_{c}$. Note that even though the absolute instability region via the third-order scattering is relatively larger than that via the second-order scattering, its intensity is much weaker. One finds a common region $[0.0764,0.11] n_{c}$ for both the second-order and third-order absolute instability modes.

It is worthwhile to check with the phase velocity of the excited electron plasma wave, which determines the energy of trapped electrons. Without loss of generality, the wavenumber match condition for the second-order absolute $\mathrm{SRS}$ is $k_{p 2}=k_{s 1}-k_{s 2}$. Since $k_{s 2} \approx 0$ for the absolute SRS, one obtains $k_{p 2} \approx k_{s 1}=-\sqrt{\omega_{s 1}^{2}-\omega_{p 2}^{2}} / c$. With the help of Equation (1), one finds the phase velocity of the electron plasma wave is simply

$$
v_{p h 2}=\omega_{p 2} / k_{p 2} \approx-c / \sqrt{3}=-0.58 c .
$$

The negative sign means that the phase velocity is opposite to the incident laser propagation as shown in Figure 1(a). Corresponding to this phase velocity, the electron energy is about $170 \mathrm{keV}$. Similarly one can find the phase velocity of the electron plasma wave excited via third-order absolute SRS $v_{p h 3}=\omega_{p 3} / k_{p 3} \approx c / \sqrt{3}$.

Now we discuss the qualitatively necessary conditions for the development of absolute modes besides the density scale. Generally backward SRS is much stronger than forward SRS due to its larger growth rate. However, when the backward SRS is heavily Landau damped in a very hot plasma, the absolute instabilities will be mainly induced via the rescattering of forward scattering ${ }^{[18,22]}$, which may play a role in the direct-drive scheme. Here we consider a plasma with a few $\mathrm{keV}$ where the backscattering SRS has not been heavily damped. The Landau damping of absolute SRS is weak due to the high phase velocity of the Langmuir wave. For example, assume the electron temperature is $2 \mathrm{keV}$, i.e., thermal velocity $v_{t h}=0.0626 c$, which is one ninth of the Langmuir wave phase velocity $v_{p h}=0.58 c$. The high temperature mainly reduces the saturation level of backscattering light, which acts as a damping of pump wave to develop absolute SRS. Therefore, the threshold for the development of absolute SRS induced via rescattering is mainly determined by the density scale length $L$ and incident laser intensity. As we know, the threshold for the first-order absolute SRS in inhomogeneous plasma is $a_{0} \gtrsim$ $\left(k_{0} L\right)^{-2 / 3[2]}$. We treat the backscattering light as a pump wave, and then we have $a_{s 0} \gtrsim\left(k_{s 0} L_{s}\right)^{-2 / 3}$ where $L_{s}=$ $L \omega_{s}^{2} / \omega_{0}^{2}$ and $k_{s 0}$ is the wavenumber of the first-order incident light, i.e., $k_{s 0}=k_{0}$. By normalizing $a_{s 0}$ back to the frequency of incident laser, we obtain the threshold for absolute SRS induced via second-order SRS rescattering

$$
a_{s} \gtrsim\left(\frac{\omega_{0}}{\omega_{s}}\right)^{1 / 3}\left(\frac{1}{k_{0} L}\right)^{2 / 3} .
$$

The above linear analysis only considers individual scattering at local plasma density. When considering the absolute SRS instability, waves scattered at different plasma densities with different frequencies can be strongly coupled. Let us consider the bandwidth effects of backscattering light at the near-quarter-critical density, where the mismatch of wave numbers becomes so weak. Considering that the mismatch of wave numbers approximates to zero for absolute instabilities, we can do the Fourier transform of the propagation equations of the scattered light and electron plasma wave similar to the homogeneous case. The width of the instability region in the plasma wave vector $\Delta k$ is proved to be a critical factor for the modes coupling ${ }^{[23]}$. The dispersion for SRS in homogeneous plasma is given by ${ }^{[1]}$

$$
\frac{\omega_{p}^{2} a_{0}^{2}}{4}\left(\frac{c^{2} k^{2}}{D_{e}}+1\right)\left(\frac{1}{D_{+}}+\frac{1}{D_{-}}\right)=1,
$$

where $D_{e}=\omega^{2}-\omega_{p}^{2}, D_{ \pm}=\omega^{2}-k^{2} c^{2} \pm 2\left(\omega_{0} \omega-k_{0} k c^{2}\right)$. Numerical solutions of Equation (5) at different densities with the normalized laser amplitude $a_{0}=0.01$ are plotted in Figure 2. The relation between $a_{0}$ and laser intensity $I_{0}$ is given by $a_{0}=\sqrt{I_{0}\left(\mathrm{~W} / \mathrm{cm}^{2}\right)[\lambda(\mu \mathrm{m})]^{2} / 1.37 \times 10^{18}}$. As show in Figure 2, at $n_{e}=0.24 n_{c}$, the instability regions of forward and backward SRS are well-separated in their wave vectors with almost equal width. However, when the plasma density increases to very near the quarter-critical density $n_{e}=0.2485 n_{c}$, one finds the forward and backward SRS sharing a common plasma wave. The width of the coupling instability region can be estimated as twice of the backward instability region at $n_{e}=0.2485 n_{c}$, i.e., $\Delta k c \approx 16.8 a_{0} \omega_{0}=$ $0.168 \omega_{0}$. Such a large instability region can greatly increase the coupling of light beams with different frequencies for the development of absolute SRS instabilities ${ }^{[23]}$. Therefore, in inhomogeneous plasma, even if the first-order backscattering 


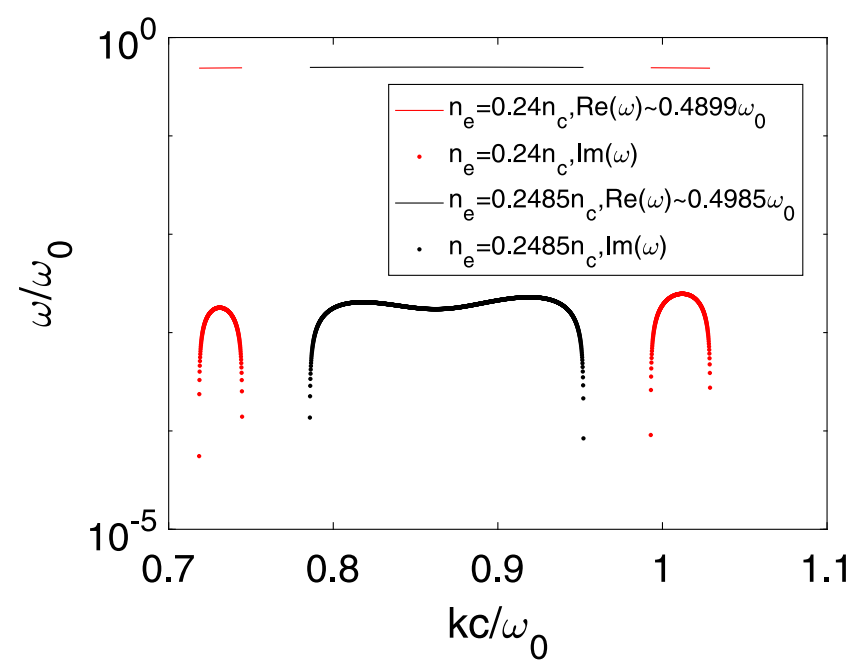

Figure 2. Numerical solutions of SRS dispersion equation at the plasma density $n_{e}=0.24 n_{c}$ and $n_{e}=0.2485 n_{c}$, where $a_{0}=0.01$. The dotted line and continuous line are the imaginary part and the real part of the solutions, respectively.

light may have broad bandwidth, they can be strongly coupled in their subsequent scattering for absolute SRS. Note that when the absolute SRS is developed, the scattered light waves have the wave number $k_{s} \approx 0$. The scattering light at the quarter-critical density can be described by near-zero refractive index photonics, which contains rich physics and extensive applications ${ }^{[24,25]}$. The trapped light with $k_{S}=0$ may be one of the reasons for laser energy deficit in inertial confinement fusion ${ }^{[20]}$. One notes that the broad width of $k$ spectrum also indicates a wide range of the Langmuir wave phase velocity, the median of which is given by Equation (3). A large range of phase velocity leads to a staged acceleration of electrons.

In conclusion, the linear analysis suggests that a laser propagating in large inhomogeneous plasma can generate a large region of absolute instabilities by cascaded scattering and coupling of different frequency components of scattering light. Both the second- and third-order rescattering of SRS contributes to the development of absolute instabilities. Even though the intensity of the third-order scattering is relatively weak, it can still be strong enough to heat abundant electrons in a long time interaction.

\section{Simulations for absolute instabilities due to the second-order rescattering of SRS}

\subsection{D simulations for absolute SRS mode}

To validate the above theoretical prediction, a series of PIC simulations has been performed in different plasma density regions by using the KLAP code ${ }^{[26]}$. First, onedimensional (1D) PIC simulations have been carried out, which avoid mixing up of various instabilities and enable one to identify the development of the multiple SRS rescattering instabilities more clearly. The space and time given in the following are normalized by the laser wavelength in vacuum $\lambda$ and the laser period $\tau$. The length of the simulation box is $700 \lambda$, where the plasma occupies a region from $25 \lambda$ to $650 \lambda$ with density profile $n_{e}(x)=0.08[1+(x-25) / 1000] n_{c}$, with $x$ the longitudinal axis. To exclude the third-order absolute mode, the minimum plasma density is set at $0.08 n_{c}$, and the maximum density is $0.13 n_{c}$. The initial electron temperature is $T_{e 0}=100 \mathrm{eV}$. The ions are immobile with a charge $Z=1$. A linearly polarized semi-infinite pump laser with a uniform amplitude $a_{0}=0.02$ (the corresponding intensity is $I_{0}=5.48 \times 10^{14} \mathrm{~W} / \mathrm{cm}^{2}$ with $\lambda=1 \mu \mathrm{m}$ ) is incident from the left boundary of the simulation box. We have taken 100 cells per wavelength and 50 particles per cell.

Based on the discussion in Section 2, a strong convective backward SRS develops in relatively high density region $n_{e} \geqslant n_{c} / 9$ due to the large growth rate, and then the produced backscattering light induces absolute SRS mode in the region $n_{e} \leqslant n_{c} / 9$. Figure 3 illustrates the development of SRS in different stages. A strong convective backscattering SRS is developed in the time window of $[1501,2000] \tau$ as shown in Figure 3(a), which is mainly caused by the first-order SRS scattering. The frequency of backscattering light ranges from $0.64 \omega_{0}$ (corresponding to $n_{1}=0.13 n_{c}$ ) to $0.72 \omega_{0}$ (corresponding to $n_{1}=0.08 n_{c}$ ). Note that the forward scattering mode is much weaker than the backward mode due to its relatively smaller growth rate, and therefore will not be discussed in the following. From Figure 3(b), we find that an absolute SRS mode has been generated during $[2001,2500] \tau$. The absolute mode is identified due to $k_{s} \approx 0$ as discussed above. The central frequency of the absolute SRS mode is around $0.32 \omega_{0}$, which is expected according to Equation (1). The wavenumber-frequency distribution of Langmuir wave is shown in Figure 3(c). The absolute mode is found at $k_{L} c=-0.56 \omega_{0}$ which is consistent with the deduction from Figure 3(b) and in agreement with Equation (3). The backscattered light (propagates from right to left) is diagnosed at $x=10 \lambda$ with its Fourier spectrum shown in Figure 3(d). A strong spectrum around $\omega_{s}=0.36 \omega_{0}$ is formed during $3001 \tau-4500 \tau$, which is produced via forward scattering of absolute SRS. The scattering spectrum can help us to identify the development of absolute SRS mode.

The generation mechanism for the absolute SRS mode can be well demonstrated in Figure 3(e). One finds a strong convective mode developed at $x=580 \lambda$ around $t=1900 \tau$, and then it gradually spread out of the resonant region. A simple calculation indicates that the quarter-critical density for the backscattering light is around $n_{e}=0.105 n_{c}$. At $t=$ $2350 \tau$, the backscattering light induces an intense absolute SRS mode near $x=320 \lambda$ where the plasma density is $n_{e}=0.104 n_{c}$. For the absolute SRS instability, the resonant region is stationary. One finds that the absolute instability region gradually widens to $[300,345] \lambda$, due to the enhanced 

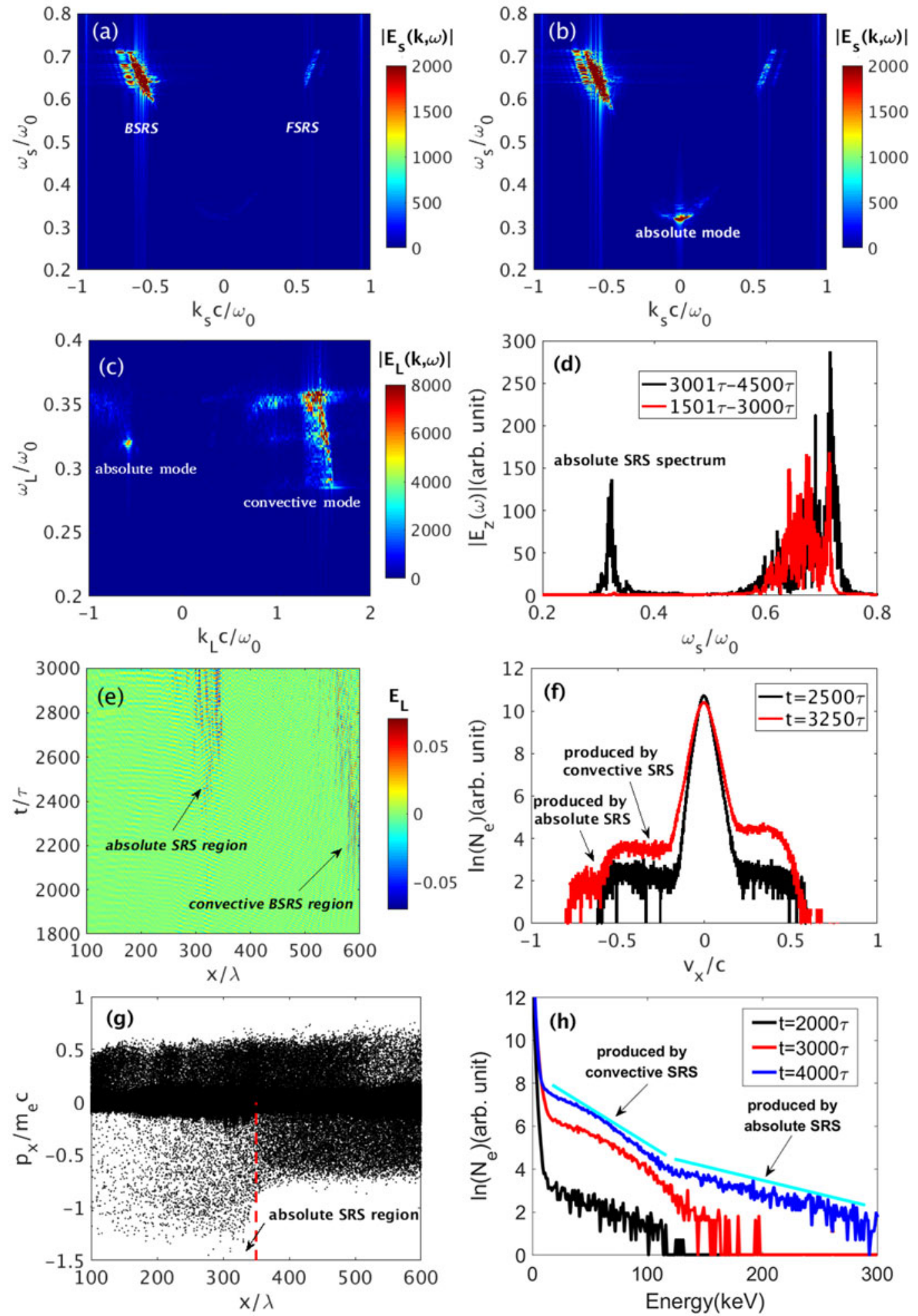

Figure 3. PIC simulation results for the development of the absolute SRS via the second-order scattering. (a) and (b) Wavenumber-frequency distributions of the scattered light in the time windows $[1501,2000] \tau$ and $[2001,2500] \tau$, respectively. FSRS means forward scattering of SRS. (c) 2D Fourier transform $\left|E_{L}(k, \omega)\right|$ of the electric field in the time window [2001, 2500] $\tau$. (d) Fourier spectra of backscattered light diagnosed at $x=10 \lambda$. (e) Time-space distributions of Langmuir waves, where $E_{L}$ is the longitudinal electric field normalized by $m_{e} \omega_{0} c / e, m_{e}, c$ and $e$ are electron mass, light speed in vacuum and electron charge, respectively. (f) Longitudinal velocity distributions of electron at different time. (g) Longitudinal phase space distribution of electrons near the region of the absolute SRS instability at $t=3250 \tau$. (h) Energy distributions of electrons at different time, where $N_{e}$ is the relative electron number. 

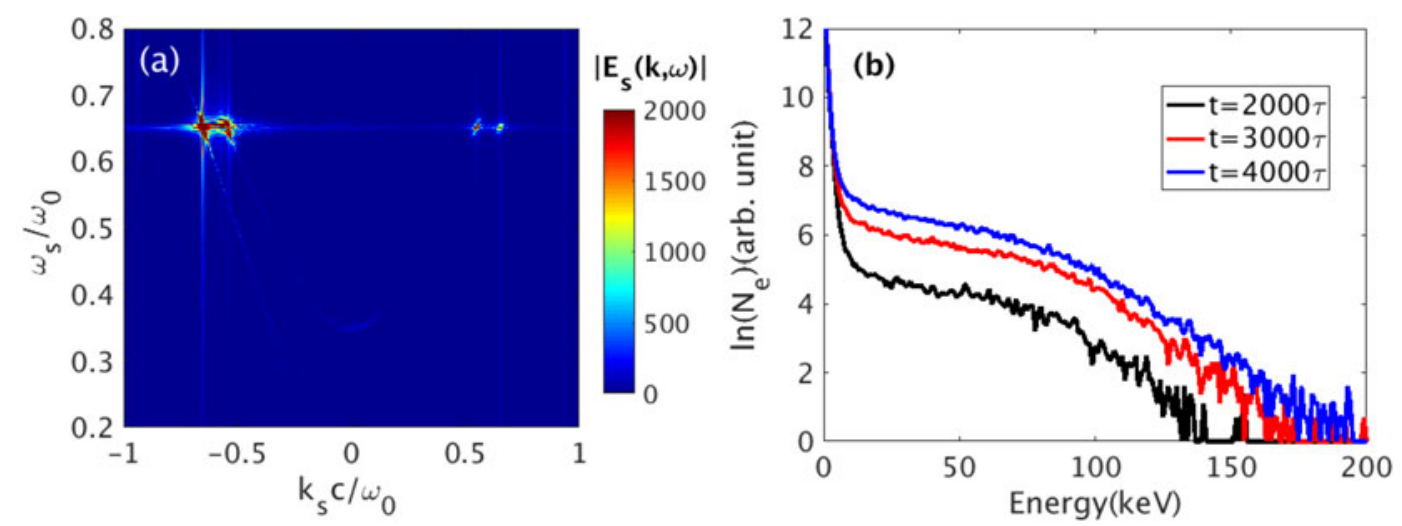

Figure 4. The case when the absolute SRS is absent provided $n_{\min }>n_{c} / 9$. (a) Wavenumber-frequency distributions of the scattered light in the time window $[2001,2500] \tau$. (b) Energy distributions of electrons at different time.

bandwidth of the backscattering light. As mentioned above, a broad instability region near the quarter-critical density can greatly reduce the suppression effects of finite bandwidth of the backscattering light. After $t=2800 \tau$, the absolute SRS becomes the dominant instability mode.

According to Equation (3), the phase velocity of the Langmuir wave excited by the absolute SRS is very high. In linear stage, the temperatures of most electrons are much lower than $170 \mathrm{keV}$. Therefore, the absolute SRS mode can grow without Landau damping. The main damping for the growth of absolute SRS mode comes from the backscattering light developed via backward SRS. In our simulation, the phase velocity of the Langmuir wave developed by convective SRS is around $0.26 c$. From Figure 3(f), we find that the electron energy distribution is flattened around $v_{x}=-0.27 c$ by trapped electrons at $t=2500 \tau$. Note that the velocity of most electrons is less than $0.58 c$. Therefore, this time period is the linear stage for the absolute SRS instability. At $t=3250 \tau$, the absolute SRS mode has started evolving into the nonlinear regime, and another flattened region near $v_{x}=$ $-0.6 c$ is formed. Figure $3(\mathrm{~g})$ presents a clear physical picture of electrons accelerated in the absolute SRS region. The maximum electron momentums are below $\left|p_{x}\right| \sim 0.7 m_{e} c$ at $x>350 \lambda$. When the electrons propagate into the absolute SRS region, they are accelerated up to even $\left|p_{x}\right| \sim 1.5 m_{e} c$.

The overall electron energy distribution has been diagnosed as shown in Figure 3(h). Before the development of the absolute SRS, electron heating is weak at $t=2000 \tau$. During $2000 \tau$ to $3000 \tau$, however, a large number of hot electrons start to be heated up to above $100 \mathrm{keV}$. Afterwards when the process evolves into the nonlinear regime, lots of hot electrons can be trapped by the Langmuir wave and heated even up to $300 \mathrm{keV}$, where a hot electron tail with temperature around $T_{e}=78 \mathrm{keV}$ is generated by the absolute SRS mode at $t=4000 \tau$. One finds that absolute SRS plays a leading role in the heating process after $t=2600 \tau$. It has been reported that experiments in NIF demonstrate that the electrons with temperature $T_{e}>170 \mathrm{keV}$ can cause ignition capsule preheat ${ }^{[13]}$. Therefore, the absolute SRS instability induced by convective backward SRS could be a crucial factor in the long time interactions.

To further validate the theoretical predictions on the condition of absolute SRS instability, we have performed a simulation with plasma density profile $n_{e}(x)=0.12[1+(x-$ $525) / 1500] n_{c}$ with plasma region $[525,650] \lambda$, where the minimum density is larger than $n_{c} / 9$. The other parameters are the same as the above simulation. As discussed in Section 2, under this plasma condition, the second-order absolute SRS mode would not be triggered. As shown in Figure 4(a), only convective SRS mode can be found in the phase distribution of scattered light in the same time window with Figure 3(b), i.e., no absolute SRS is developed. The absolute SRS mode is thought to be a mechanism for the production of hot electrons. As shown in Figure 4(b), the electron energy distribution tends to be saturated after $t=3000 \tau$, and the maximum electron energy is still less than $200 \mathrm{keV}$ at $t=4000 \tau$. This simulation result further proves that hot electron production is considerably reduced if no absolute SRS mode is developed.

Based upon the above two simulations as well as the simulation with density range $[0.08,0.1] n_{c}$, we calculate the energy ratio of electrons heated by absolute SRS to the whole electrons with energy $>60 \mathrm{keV}$ when the electron temperatures are saturated, and the result is $\sim 30 \%$. This calculation indicates that absolute instabilities in inhomogeneous plasma have a crucial effect on the production of hot electrons.

Now we study the absolute SRS developed in a plasma at temperature $T_{e 0} \sim \mathrm{keV}$. First, the simulation has been performed at $T_{e 0}=1 \mathrm{keV}$. The other parameters are the same as in the first simulation example. An absolute SRS mode with finite bandwidth can be found around $k_{L} c=-0.6 \omega_{0}$ and $\omega_{L}=0.33 \omega_{0}$ in the time window [2001,2500] $\tau$ as shown in Figure 5(a). The low-frequency convective mode is much weaker than the case with $T_{e 0}=100 \mathrm{eV}$, due to the heavy Landau damping. Note that the characteristic collision time for $1 \mathrm{keV}$ plasma is larger than $10^{4} \tau$; therefore, the 

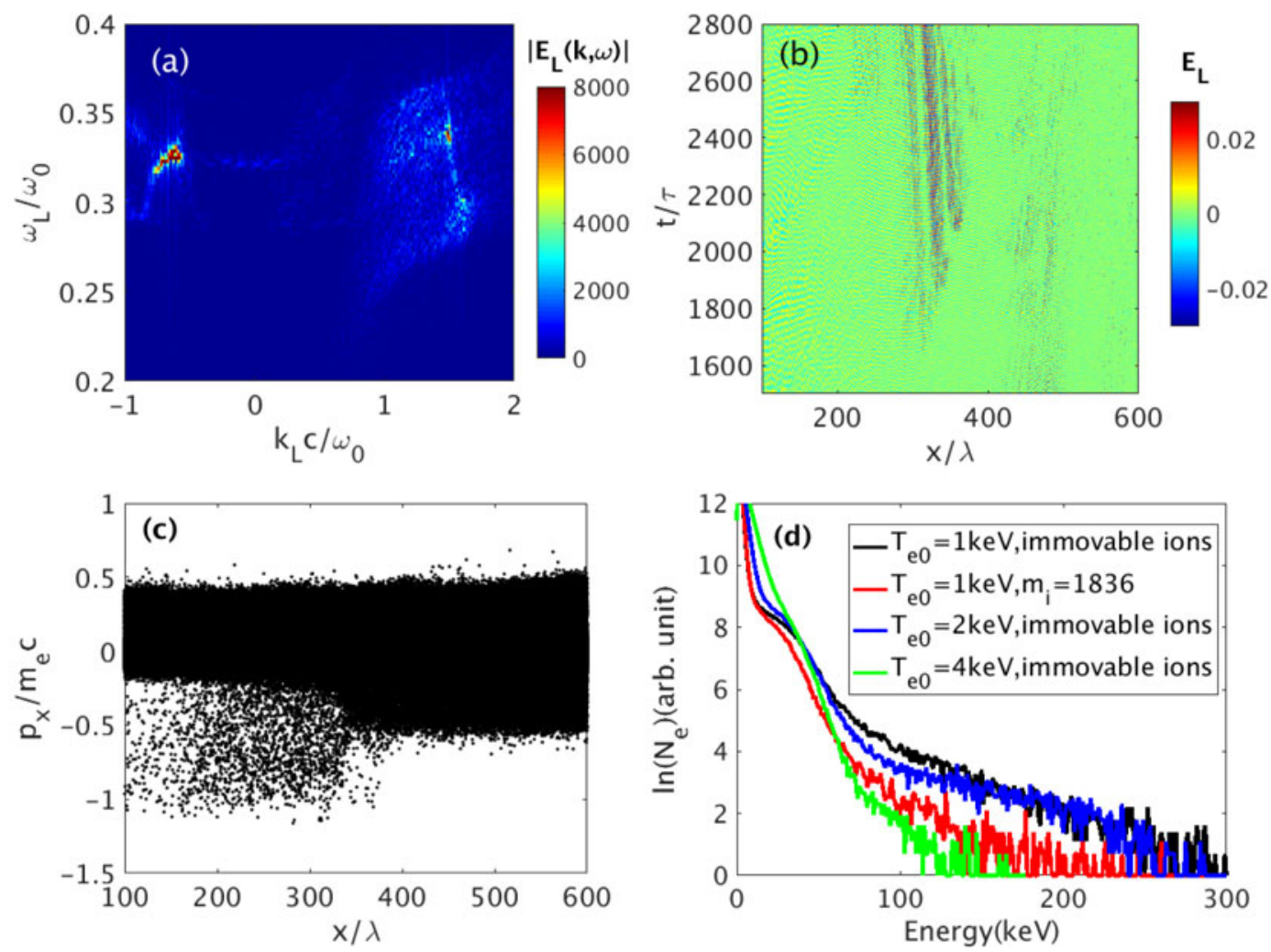

Figure 5. (a)-(c) PIC simulation results for a plasma at $T_{e 0}=1 \mathrm{keV}$ with immovable ions. (a) $2 \mathrm{D}$ Fourier transform $\left|E_{L}(k, \omega)\right|$ of the electric field in the time window [2001, 2500] $\tau$. (b) Time-space distributions of Langmuir waves. (c) Longitudinal phase space distribution of electrons at $t=2800 \tau$. (d) Energy distributions of electrons with different temperatures or different ions at $t=4000 \tau$.

collisions have weak effects on the absolute SRS. Figure 5(b) provides the time-space distributions of Langmuir waves. The absolute SRS mode develops around $x \sim 330 \lambda$ at $t=$ $1900 \tau$, and its intensity is much stronger than the convective backward SRS mode. The longitudinal momentum distributions displayed in Figure 5(c) indicate that the hot electrons can be accelerated up to $\left|p_{x}\right| \sim 1 m_{e} c$ when they transport into absolute instability region $\sim 330 \lambda$. The above results demonstrate that the absolute SRS can be developed even at keV plasma.

To investigate the effects of electron temperature and ion acoustic wave on the absolute SRS, we performed other three simulations as shown in Figure 5(d). The hot tail is barely reduced when the electron temperature increases from $1 \mathrm{keV}$ to $2 \mathrm{keV}$. Considerable suppression is found when $T_{e 0}=$ $4 \mathrm{keV}$; however, the hot tail still exists. The hot tail with movable ions $m_{i}=1836$ at electron temperature $T_{e 0}=1 \mathrm{keV}$ and ion temperature $T_{i 0}=0.76 \mathrm{keV}$ is obviously reduced as compared to the case with stationary ions. Ion acoustic wave always weakens the strength of convective SRS by triggering stimulated Brillouin scattering (SBS), Langmuir decay instability (LDI) and plasma density modulations ${ }^{[18]}$. However, the ion effects are weakened at keV plasmas, due to the reduction of ion acoustic wave ${ }^{[8,27,28]}$. In conclusion, we can find a relatively weak absolute SRS mode in the plasma with keV electron temperature and movable ions.

\section{2. $2 D$ simulations for absolute instability modes}

To further validate the absolute instabilities induced via the rescattering of SRS in high dimension, we performed a 2D PIC simulation. The length of the simulation box is $550 \lambda$, where the plasma occupies a region from $25 \lambda$ to $525 \lambda$ with density profile $n_{e}(x)=0.0998[1+(x-25) / 2000] n_{c}$. The width of the plasma is $20 \lambda$. The initial electron temperature is $T_{e 0}=2 \mathrm{keV}$. The ions are immovable with a charge $Z=1$. A p-polarized (electric field of light is parallel to the simulation plane) semi-infinite pump laser with a uniform amplitude $a_{0}=0.02$ (the corresponding intensity is $I_{0}=$ $5.48 \times 10^{14} \mathrm{~W} / \mathrm{cm}^{2}$ with $\lambda=1 \mu \mathrm{m}$ ) is incident from the left boundary of the simulation box. We have taken 50 cells per wavelength in both transverse and longitudinal directions.

Figure 6(a) exhibits the spatial Fourier spectra of longitudinal electric field. An intense absolute SRS mode has been developed near $k_{x} c=0.58 \omega_{0}$ at $t=2300 \tau$, which confirms that the absolute SRS is not only a one-dimensional effect. The TPD mode has not been found in this region due to high electron temperature. As known, the ratio between 

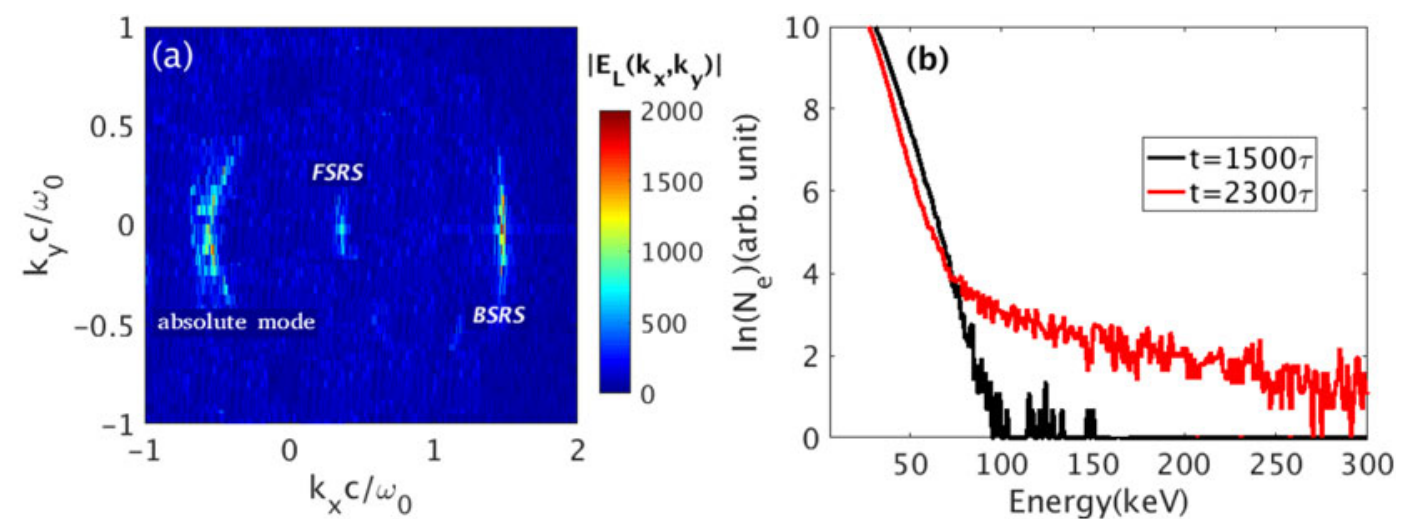

Figure 6. Development of absolute SRS instability as seen from 2D PIC simulation with $a_{0}=0.02$ and $T_{e 0}=2 \mathrm{keV}$. The incident laser is p-polarized. (a) Spatial Fourier transform $\left|E_{L}\left(k_{x}, k_{y}\right)\right|$ of the electric field at $t=2300 \tau$. (b) Energy distributions of electrons at different time.

the thresholds for SRS and TPD is roughly $\left(24 / T_{e 0}\right) / L^{1 / 3}$; therefore SRS-dominated regime can be separated from TPD-dominant regime via increasing electron temperature in a finite-size inhomogeneous plasma ${ }^{[8]}$. The energy spectrum of hot electrons is shown in Figure 6(b), where a hot electron tail is formed at $t=2300 \tau$. This further proves that the absolute instabilities induced via SRS rescattering do contribute significantly to the hot electron productions.

\section{Simulations for absolute SRS mode via the third- order rescattering of SRS}

The above simulations are calculated for absolute SRS mode via the second-order rescattering of SRS. It is possible that absolute SRS mode via the third-order scattering can be found in a large scale plasma according to our theoretical analysis. In this section we show 1D simulation results to demonstrate this. An inhomogeneous plasma with density profile $n_{e}(x)=0.04[1+(x-25) / 1000] n_{c}$ occupies a region from $x=25 \lambda$ to $x=1275 \lambda$. Note that the plasma density range covers $n_{e}=0.0625 n_{c}$, and the maximum plasma density is $n_{\max }=0.09 n_{c}<n_{c} / 9$. The initial electron temperature is $T_{e 0}=100 \mathrm{eV}$. The ions are stationary with a charge $Z=1$. The incident laser amplitude is $a_{0}=0.02$.

Figure 7 illustrates the evolution of the scattering waves and Langmuir waves in the wavenumber-frequency space. Figure 7(a) shows clearly the cascaded scattering has developed, including the first-order scattering, the second-order scattering and even the third-order scattering. It also suggests that the third-order scattering is the convective backward SRS mode, which is generated around $t=4000 \tau$ at a very low intensity. Some time later, a relatively strong absolute mode can be found in the time window $[4001,5000] \tau$ as shown in Figure 7(b). The absolute instability mode can be clearly distinguished from the convective one in the spectrum distribution. One notes that the absolute mode is developed in the density region ranging from $0.066 n_{c}$ to $0.077 n_{c}$ in this period.
Figure 7(c) shows the distribution of the Langmuir wave in $\left(k_{L}, \omega_{L}\right)$ space. The white line marks the linear resonant region for the convective backward SRS obtained from $k_{L} c=\sqrt{\omega_{0}^{2}-\omega_{L}^{2}}+\sqrt{\omega_{0}^{2}-2 \omega_{0} \omega_{L}}$. Broad wave number spectrum indicates that the convective modes spread in space with strong frequency shift ${ }^{[29]}$. Moreover, the generation of the third-order backward SRS and the increasing of electron temperature can also lead to the wave number shift. The absolute mode around $k_{L}=0.4 \omega_{0} / c$ with bandwidth $0.022 \omega_{0}$ demonstrates that the absolute SRS instability region is $[0.067,0.079] n_{c}$, which is the same as the range inferred from Figure 7(b). One notes that the intensity of the absolute SRS mode becomes comparable to the firstorder backward SRS at $6000 \tau$. When the absolute SRS mode induced by third-order rescattering of SRS suffers Landau damping, then the Langmuir wave will transfer energy to the hot electrons.

As comparison, we have performed another set of simulation with a plasma density profile limited to the range $[0.0625,0.09] n_{c}$ and other parameters unchanged. Based on the discussion in Section 2, the development of the absolute SRS mode via the third-order scattering needs a secondorder backward SRS region $n_{e}<0.0625 n_{c}$ to generate backscattering lights. Without this density region, only a convective mode can be found and no third-order absolute SRS can be developed. This is verified in Figure 7(d).

\section{Summary}

In summary, we have theoretically and numerically studied the mechanism of the absolute instabilities induced by cascading SRS processes in a large scale nonuniform plasma. Theoretical analysis indicates that the backscattering light from convective SRS can generate strong absolute instabilities in a plasma within $n_{e}<1 / 9 n_{c}$ via the secondorder SRS rescattering. The absolute SRS mode develops a Langmuir wave with a high phase velocity of about 

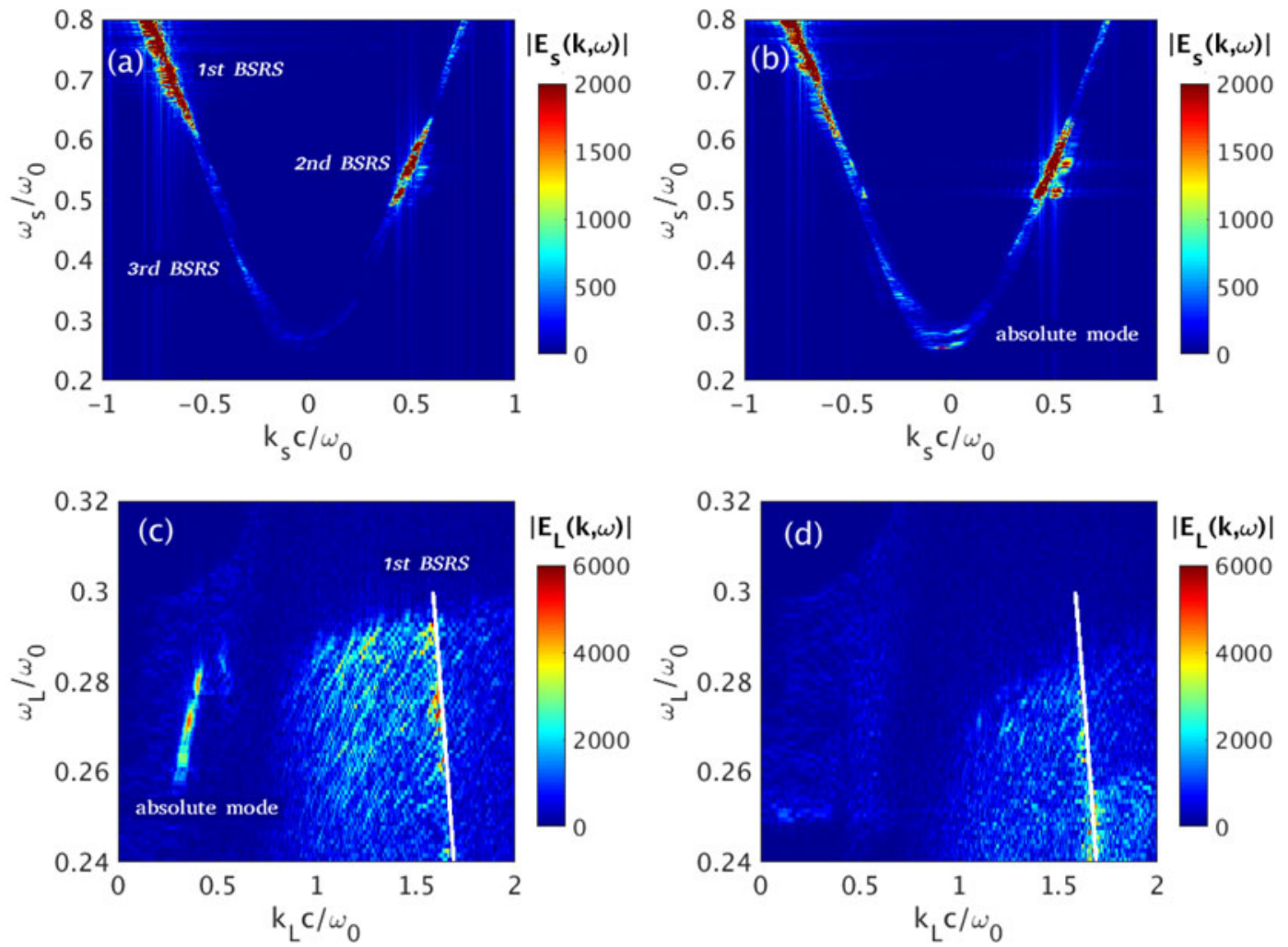

Figure 7. PIC simulation results for the development of the absolute SRS via the third-order scattering. (a)-(c) Simulation results for the plasma with the inhomogeneous plasma density range $[0.04,0.09] n_{c}$. (a) and (b) show the $2 \mathrm{D}$ Fourier transform $\left|E_{S}(k, \omega)\right|$ of the scattered light $E_{S}(x, t)$ in the time windows $[3001,4000] \tau$ and $[4001,5000] \tau$, respectively. (c) 2D Fourier transform $\left|E_{L}(k, \omega)\right|$ of the electric field in the time window [5001, 6000] $\tau$. The white line denotes the linear resonant region for convective backscattering SRS. (d) 2D Fourier transform $\left|E_{L}(k, \omega)\right|$ of the electric field in the time window $[5001,6000] \tau$, when the plasma density profile is limited to the range of $[0.0625,0.09] n_{c}$.

$0.58 c$, corresponding to hot electron energy around $170 \mathrm{keV}$. In the linear stage of the absolute SRS instability, the absolute SRS mode can grow without Landau damping, where the hot electron energy is well below $100 \mathrm{keV}$. When the interactions evolve into the nonlinear regime, due to the rescattering processes, a large number of electrons are trapped by the Langmuir wave and are heated up to $300 \mathrm{keV}$. It is further demonstrated that the absolute SRS instability can be developed via the third-order backward SRS when the inhomogeneous plasma is even larger and the interaction time is long over a few picoseconds. The absolute instabilities due to rescattering of SRS may play a significant role for the capsule preheating in inertial confinement fusion, where a large inhomogeneous plasma is often involved.

\section{Acknowledgements}

The authors acknowledge useful discussions with C. Z. Xiao, R. Yan and C. Ren. This work was supported by the National Natural Science Foundation of China (Nos. 11775144 and 1172109) and the Natural Science Foundation of Shanghai (No. 19YF1453200).

\section{References}

1. W. L. Kruer, The Physics of Laser Plasma Interactions (Addison-Wesley, New York, 1988).

2. C. Liu, M. N. Rosenbluth, and R. B. White, Phys. Fluids 17, 1211 (1974).

3. D. S. Montgomery, Phys. Plasmas 23, 055601 (2016).

4. R. Betti and O. A. Hurricane, Nature Phys. 12, 435 (2016).

5. J. Lindl, O. Landen, J. Edwards, E. Moses, and N. Team, Phys. Plasmas 21, 020501 (2014).

6. O. A. Hurricane, D. A. Callahan, D. T. Casey, P. M. Celliers, C. Cerjan, E. L. Dewald, T. R. Dittrich, T. Döppner, D. E. Hinkel, and L. F. Berzak Hopkins, Nature 506, 343 (2014).

7. E. M. Campbell, V. N. Goncharov, T. C. Sangster, S. P. Regan, P. B. Radha, R. Betti, J. F. Myatt, D. H. Froula, M. J. Rosenberg, and I. V. Igumenshchev, Matter Radiat. Extrem. 2, 37 (2017).

8. S. Weber and C. Riconda, High Power Laser Sci. Eng. 3, e51 (2015).

9. X. T. He, J. W. Li, Z. F. Fan, L. F. Wang, J. Liu, K. Lan, J. F. Wu, and W. H. Ye, Phys. Plasmas 23, 082706 (2016).

10. V. A. Smalyuk, D. Shvarts, R. Betti, J. A. Delettrez, D. H. Edgell, V. Y. Glebov, V. N. Goncharov, R. L. McCrory, D. D. Meyerhofer, P. B. Radha, S. P. Regan, T. C. Sangster, W. Seka, S. Skupsky, C. Stoeckl, B. Yaakobi, J. A. Frenje, C. K. Li, R. D. Petrasso, and F. H. Séguin, Phys. Rev. Lett. 100, 185005 (2008). 
11. T. C. Sangster, V. N. Goncharov, P. B. Radha, V. A. Smalyuk, R. Betti, R. S. Craxton, J. A. Delettrez, D. H. Edgell, V. Y. Glebov, D. R. Harding, D. Jacobs-Perkins, J. P. Knauer, F. J. Marshall, R. L. McCrory, P. W. McKenty, D. D. Meyerhofer, S. P. Regan, W. Seka, R. W. Short, S. Skupsky, J. M. Soures, C. Stoeckl, B. Yaakobi, D. Shvarts, J. A. Frenje, C. K. Li, R. D. Petrasso, and F. H. Séguin, Phys. Rev. Lett. 100, 185006 (2008).

12. D. Batani, S. Baton, A. Casner, S. Depierreux, M. Hohenberger, O. Klimo, M. Koenig, C. Labaune, X. Ribeyre, C. Rousseaux, G. Schurtz, W. Theobald, and V. Tikhonchuk, Nucl. Fusion 54, 054009 (2014).

13. E. L. Dewald, C. Thomas, S. Hunter, L. Divol, N. Meezan, S. H. Glenzer, L. J. Suter, E. Bond, J. L. Kline, J. Celeste, D. Bradley, P. Bell, R. L. Kauffman, J. Kilkenny, and O. L. Landen, Rev. Sci. Instrum. 81, 10D938 (2010).

14. S. P. Regan, N. B. Meezan, L. J. Suter, D. J. Strozzi, W. L. Kruer, D. Meeker, S. H. Glenzer, W. Seka, C. Stoeckl, and V. Y. Glebov, Phys. Plasmas 17, 055503 (2010).

15. D. J. Strozzi, E. A. Williams, D. E. Hinkel, D. H. Froula, R. A. London, and D. A. Callahan, Phys. Plasmas 15, 102703 (2008).

16. B. Winjum, J. Fahlen, F. Tsung, and W. Mori, Phys. Rev. Lett. 110, 165001 (2013).
17. W. L. Kruer, K. Estabrook, B. F. Lasinski, and A. B. Langdon, Phys. Fluids 23, 1326 (1980).

18. D. E. Hinkel, S. W. Haan, A. B. Langdon, T. R. Dittrich, C. H. Still, and M. M. Marinak, Phys. Plasmas 11, 1128 (2004).

19. K. Mima, M. S. Jovanović, Y. Sentoku, Z. M. Sheng, M. M. Skoric, and T. Sato, Phys. Plasmas 8, 2349 (2001).

20. O. Klimo, S. Weber, V. T. Tikhonchuk, and J. Limpouch, Plasma Phys. Control. Fusion 52, 055013 (2010).

21. J. Myatt, J. Zhang, R. Short, A. Maximov, W. Seka, D. Froula, D. Edgell, D. Michel, I. Igumenshchev, D. Hinkel, P. Michel, and J. D. Moody, Phys. Plasmas 21, 055501 (2014).

22. A. B. Langdon and D. E. Hinkel, Phys. Rev. Lett. 89, 015003 (2002).

23. Y. Zhao, S. Weng, M. Chen, J. Zheng, H. Zhuo, C. Ren, Z. Sheng, and J. Zhang, Phys. Plasmas 24, 112102 (2017a).

24. I. Liberal and N. Engheta, Nature Photon. 11, 149 (2017).

25. M. M. Ahmed and N. Nader, Nature Commun. 5, 5638 (2014).

26. M. Chen, Z. M. Sheng, J. Zheng, Y. Y. Ma, and J. Zhang, Chin. J. Comput. Phys. 25, 43 (2008).

27. S. Weber, C. Riconda, and V. T. Tikhonchuk, Phys. Rev. Lett. 94, 055005 (2005).

28. Y. Zhao, L. L. Yu, S. M. Weng, C. Ren, C. S. Liu, and Z. M. Sheng, Phys. Plasmas 24, 092116 (2017).

29. B. Winjum, J. Fahlen, and W. Mori, Phys. Plasmas 14, 102104 (2007). 